\section{Sofortige Operation bei erworbenem Hodenhochstand nicht nötig}

\author{
Wird bei Knaben ein erworbener Hodenhochstand erkannt, muss nicht \\ gleich der Chirurg aktiv werden. Eine rasche Orchidopexie verbessert die \\ Fruchtbarkeit nicht unbedingt.
}

$B^{\text {e }}$ ei kongenitalem Maldescensus testis wird meist noch vor Ende des ersten Lebensjahres eine Operation empfohlen, wenn die Hoden bis dahin nicht von allein oder mithilfe einer Hormontherapie ihre natürliche Lage gefunden haben. Damit soll die Fruchtbarkeit verbessert und das Krebsrisiko gesenkt werden.

Noch keinen klaren Konsens gibt es jedoch bei der Frage, welche Therapiestrategien am besten sind, wenn sich ein Hodenhochstand erst im Laufe der Entwicklung des Kindes einstellt. Einige Urologen plädieren dann ebenfalls für eine schnelle Orchidopexie, andere wollen erst einmal abwarten, ob sich die Hoden bis zur Pubertät wieder ins Skrotum zurückziehen. Ein solches Wait-and-See-Protokoll wird inzwischen in den Niederlanden empfohlen, berichten Evelyn van der Plas und Kollegen von der Erasmus-Universität in Rotterdam.
$\mathrm{Ob}$ „wait and see“ tatsächlich keine Nachteile bringt, haben die Forscher nun anhand einer Analyse bei 128 jungen Männern geprüft, bei denen zwischen 1982 und 2009 ein erworbener Hodenhochstand festgestellt wurde. Die Männer waren bei der Analyse im Schnitt rund 28 Jahre alt. Bei 65 von ihnen hatten sich die Ärzte zuvor fürs Abwarten entschieden. Eine Operation sollte nur erfolgen, wenn die Hoden bis zum Tannerstadium P2G2 nicht wieder abgestiegen waren - das war bei rund der Hälfte der Fall. Bei 15 der Beobachteten wurde ein beidseitiger Hodenhochstand festgestellt, auch hier kam es bei etwa der Hälfte zu einem spontanen Abstieg. Wurde operiert, so erfolgte dieser Eingriff im Schnitt im Alter von 13,2 Jahren.

Bei 63 der Männer hatten sich die Ärzte für eine Orchidopexie unmittelbar nach der Diagnose entschieden. Wurden nun die Männer mit einstigem

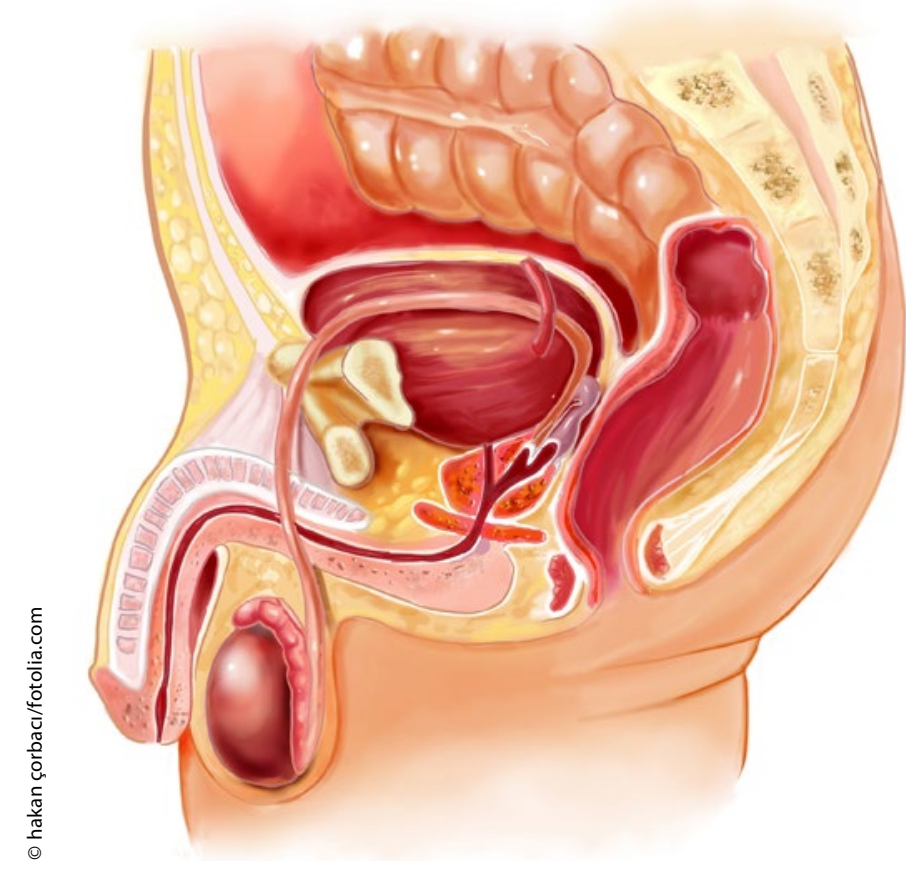

unilateralem Hodenhochstand verglichen, so gab es kaum signifikante Unterschiede bei der Hodenfunktion zwischen denen mit sofortiger Orchidopexie und denjenigen mit abwartender Strategie: Das Hodenvolumen war vergleichbar (8,6 vs. 9,3 ml), ebenso die Spermienkonzentration (20 vs 22 Mio./ $\mathrm{ml}$ ) und auch die Konzentration von Hormonen wie LH, FSH und Testosteron. Mit sieben versus vier Vaterschaften konnten die Männer in der Gruppe mit abwartender Strategie etwas mehr Kinder zeugen, dafür trat eine Oligozoospermie bei ihnen etwas häufiger auf (38 vs. $27 \%$ ), und eine Asthenospermie war doppelt so oft zu beobachten (22 vs. $11 \%)$. All diese Unterschiede waren jedoch nicht signifikant. Statistisch belastbar waren in der Gruppe mit abwartender Strategie die Inhibin-B-Werte erhöht (161 vs. $134 \mathrm{ng} / \mathrm{l}$ ), dafür war die Progressivmotilität geringer ( 45,0 vs. 47,5\%). Doch daraus lässt sich nach Angaben der Studienautoren um van der Plas wenig schließen.

Fazit: Die Ergebnisse der Untersuchungen deuteten vielmehr auf eine insgesamt reduzierte Fertilität der Männer mit Hodenhochstand hin. Daran scheine auch die frühe Operation nichts zu ändern, so das Resümee der Studienautoren. Aus diesem Grund sei hier wohl eine konservative Vorgehensweise zu empfehlen.

Etwas anders sieht es möglicherweise bei Männern mit beidseitigem Hodenhochstand aus $(\mathrm{n}=29)$. Bei ihnen sprechen einige Fruchtbarkeitsparameter für eine rasche Orchidopexie: So gab es bei den 14 sofort Operierten mehr Vaterschaften (6 vs. 1), die Spermienkonzentration war höher (22 vs. $8 \mathrm{Mio} . / \mathrm{ml})$, und auch die Progressivmotilität (45 vs. 25\%). Entsprechend war der Anteil der Männer mit Asthenospermie geringer (23 vs. $57 \%$ ). Statistisch belastbar waren die Unterschiede jedoch nicht - dafür war wohl auch die Zahl der Teilnehmer zu klein.

Thomas Müller

\author{
Der Hoden in \\ seiner korrekten \\ Lage: was tun, \\ wenn es anders ist?
}

Van der Plas EM et al. Acquired undescended testes and fertility potential: is orchiopexy at diagnosis better than awaiting spontaneous descent? Andrology. 2015; 3(4):677-84. 